\title{
Capillary LC-MS for High Sensitivity Metabolomic Analysis of Single Islets of Langerhans
}

\author{
Qihui Ni ${ }^{\dagger}$, Kendra R. Reid ${ }^{\dagger}$, Charles F. Burant ${ }^{\ddagger}$, and Robert T. Kennedy $\dagger$,§ \\ The University of Michigan, Department of Chemistry, Department of Internal Medicine and \\ Department of Pharmacology, Ann Arbor, Michigan 48109
}

\section{Abstract}

Reversed-phase, packed capillary liquid chromatography interfaced by electrospray ionization to mass spectrometry was explored as an analytical method for determination of metabolites in microscale tissue samples using single islets of Langerhans as a model system. Using a $75 \mu \mathrm{m}$ inner diameter column coupled to a quadrupole ion trap mass spectrometer in full scan mode, detection limits of 0.1 to $33 \mathrm{fmol}$ were achieved for glycoloytic and tricarboxylic acid cycle metabolites. Reproducible processing of islets for analysis with little loss of metabolites was performed by rapid freezing followed by methanol: water extraction. The method yielded $20 \mu \mathrm{L}$ of extract of which just $15 \mathrm{~nL}$ was injected suggesting the potential for performing multiple assays on the same islet. Approximately 200 presumed metabolites could be detected, of which 22 were identified by matching retention times and MS/MS spectra to standards. Relative standard deviations for peak detection was from 7 to $18 \%$ and was unaffected by storage for up to 11 days. The method was used to detect changes in metabolism associated increasing extracellular islet glucose concentration from 3 to 20 $\mathrm{mM}$ yielding results largely consistent with known metabolism of islets. Because most previous studies of islet metabolism have only observed a few compounds at once and require far more tissue, this measurement method represents a significant advance for studies of metabolism of islets and other microscale samples.

\section{Introduction}

Metabolomics is becoming an increasingly important tool in biology. Wide scale measurement of metabolites has found use in determination of biochemical signaling pathways, gene function, drug effects, and for metabolic engineering. ${ }^{1-4}$ The analytical tools used for such studies have included direct infusion mass spectrometry (MS), GC-MS, HPLC-MS, capillary electrophoresis (CE)-MS, FT-IR and NMR. ${ }^{2,}$ 5-9 Most studies have not been concerned with microscale samples; therefore, little effort has been made to miniaturize metabolomic analysis. This lack of miniaturization is likely due to metabolomics being largely driven by analysis of plants, microbes, and bodily fluids. In such cases, samples are relatively large and mass sensitivity is not a concern. It is reasonable to expect however that as the utility of metabolomics grows, it will be used in cases where samples are limited. One can envision that metabolomic analysis of highly heterogeneous tissue, such as brain, inherently small samples such as embryos, or difficult to harvest tissues will be of interest. For such cases, it will become important to develop methods with sufficient sensitivity. Direct MALDI-MS of tissues has shown compatibility with small samples ${ }^{10}$; however, techniques that can detect more

Corresponding Author: Robert Kennedy, 930 N. University Ave., Department of Chemistry, University of Michigan, Ann Arbor, MI 48109-1055, Telephone: (734)615-4363, Fax: (734) 615-6462, E-mail: rtkenn@umich.edu.

Department of Chemistry

\$Department of Internal Medicine

$\S$ Department of Pharmacology 
compounds are required. It is well-known that miniaturization of chromatography columns will improve mass sensitivity due to decreased dilution of a given mass injected. 11,12 Miniaturization is also important for interface to electrospray ionization (ESI)-MS analysis because of improved ionization efficiency from small electrospray emitters operated at low flow rates. ${ }^{13}$ Because of these effects, low attomole detection limits have been achieved for select compounds using capillary LC-ESI-MS-MS. ${ }^{14}$

In this work, we have examined the use of capillary LC-quadrupole ion trap (QIT)-MS as an analytical method for determination of metabolites in single islets of Langerhans, which are microorgans found in the pancreas that contain a few thousand cells each. We demonstrate that detection limits of 0.1 to $33 \mathrm{fmol}$ for polar anions in $15 \mathrm{~nL}$ injection volumes (corresponding to $7 \mathrm{nM}$ to $2 \mu \mathrm{M}$ ) can be achieved using a $75 \mu \mathrm{m}$ inner diameter column coupled to a QIT-MS operated in full scan mode. With these detection limits, it is possible to reproducibly process a single islet of Langerhans and detect $\sim 200$ metabolites using $0.1 \%$ of the islet sample corresponding to $\sim 2$ cells. These results demonstrate that capillary LC-MS will be useful for sample-limited metabolomic measurements.

Islets are a useful model system for this study because of the importance of metabolism in their function and the need for microscale analysis. $\beta$-cells, which make up $\sim 80 \%$ of the cells of the islet, rapidly increase insulin secretion in response to elevated glucose concentration. The coupling between extracellular glucose and insulin secretion involves alterations in metabolism such that changes in intracellular metabolite concentration result in signals that evoke exocytosis of insulin. Although increase in the ATP/ADP ratio is a well-established metabolic signal, ${ }^{15}$ it is generally believed that other secretory signals exist. ${ }^{16,17}$ Impaired insulin secretion 18 and apoptotic reduction of $\beta$-cell mass associated with type 2 diabetes are believed to involve alteration in $\beta$-cell metabolism. Efforts in metabolomic analysis of islets or related cell lines have already begun, though these studies have been performed using relatively large samples. ${ }^{19,20}$ Islet isolation from small rodents only yields 50-125 islets. While such islets may be pooled for analysis, it would be useful to analyze islets from a single experimental animal under different in vitro conditions. Furthermore, islets are known to be heterogeneous in their function and significant insights into islet heterogeneity could be gleaned from the study of individual islets. Our study illustrates that such studies are possible using the method described here.

\section{Experimental}

\section{Reagents and Materials}

All chemicals were purchased from Sigma-Aldrich (St Louis, MO) unless otherwise stated. All solvents were purchased from Burdick \& Jackson (Muskegon, MI). RPMI media, fetal bovine serum and penicillin-streptomycin were purchased from Invitrogen Corp (Carlsbad, CA). Carbon-13 enriched glucose was purchased from Cambridge Isotope (Andover, MA). Eppendorf Safelock Biopur tubes were purchased from Fisher Scientific (Fairland, NJ). Fused silica capillary was purchased from Polymicro Technologies (Phoenix, AZ). Reversed phase (RP) chromatographic stationary phase was purchased from Waters Corp (Milford, MA). Strong anion exchange (SAX) chromatographic stationary phase was donated by Dionex Corp (Sunnyvale, CA).

\section{Islet Isolation}

Islets of Langerhans were isolated from pancreas as previously described with slight modifications. ${ }^{21}$ Briefly, 20-30 g male CD-1 mice were euthanized followed by inflation of the pancrease by ductal injection of $0.5 \mathrm{mg} / \mathrm{mL}$ collagenase XI. The pancreas was dissected and digested by $5 \mathrm{~mL}$ of $0.5 \mathrm{mg} / \mathrm{mL}$ collagenase solution at $37^{\circ} \mathrm{C}$ for $8 \mathrm{~min}$. Islets larger than 
$100 \mu \mathrm{m}$ were separated from other tissues by passing the pancreatic digestthrough a $100 \mu \mathrm{m}$ pore diameter nylon cell strainer (Fisher Scientific) with $10 \mathrm{~mL}$ of Krebs ringer buffer (KRB). Isolated islets were transferred by pipette into a Petri dish containing RPMI 1640 cell culture media with $11 \mathrm{mM}$ glucose and supplemented with $10 \%$ fetal bovine serum, 100 units $/ \mathrm{mL}$ penicillin, and $100 \mathrm{mg} / \mathrm{mL}$ streptomycin and incubated at $37^{\circ} \mathrm{C}, 5 \% \mathrm{CO}_{2}$ for 3 to 5 days before extraction.

For glucose stimulation experiments, batches of 20 islets were placed in KRB containing 3 $\mathrm{mM}$ and $20 \mathrm{mM}$ glucose respectively and incubated at $37^{\circ} \mathrm{C}$ for 45 minutes on the day of extraction. Single islets were then selected for extraction and analysis.

\section{Metabolite Extraction from Islets}

A single islet in $50 \mu \mathrm{L}$ of media contained in a $500 \mu \mathrm{L}$ Eppendorf biopur tube was snap frozen with liquid nitrogen and then thawed on ice. The islet was removed from media and extracted with $200 \mu \mathrm{L} 80 \%$ ice cold methanol/water $(\mathrm{MeOH} / \mathrm{H} 2 \mathrm{O})$. Ultrasonication by a sonic dismembrator (Fisher Scientific, Fairland, NJ) on power setting 3 for $30 \mathrm{~s}$ was used to help lysis. After $15 \mathrm{~min}$, the tube was centrifuged at $4{ }^{\circ} \mathrm{C}$ for $10 \mathrm{~min}$. The procedure was repeated twice using $200 \mu \mathrm{L}$ and $100 \mu \mathrm{L}$ solvent and the three supernatants were combined. Finally, the extracts were filtered by a Microcon YM-3 filter (Millipore, Bedford, MA) at $4{ }^{\circ} \mathrm{C}$, evaporated down to dryness using an Eppendorf Vacufuge Concentrator (Fisher Scientific, Fairland, NJ), stored at $-80{ }^{\circ} \mathrm{C}$ and reconstituted in $20 \mu \mathrm{L} 20 \mathrm{mM}$ formic acid before separation.

For isotope tracer spike-in tests, isolated islets were incubated directly in glucose-free RPMI 1640 cell culture media with $11 \mathrm{mM} \mathrm{U}-{ }^{13} \mathrm{C}$-labeled glucose added (supplemented with $10 \%$ fetal bovine serum, 100 units $/ \mathrm{mL}$ penicillin, and $100 \mathrm{mg} / \mathrm{mL}$ streptomycin) for at least $24 \mathrm{~h}$. Extractions of the islets were performed at the same time. A total of 6 islets were used, 3 as control, to confirm that the target metabolites were fully labeled. ${ }^{12} \mathrm{C}$ standards were spiked into the $80 \% \mathrm{MeOH} / \mathrm{H}_{2} \mathrm{O}$ extraction solution for the experimental group. The standards used were $15 \mathrm{nM}$ fructose 6-phosphate (F6P), $40 \mathrm{nM}$ fructose 1,6-bisphosphate (FBP), $30 \mathrm{nM}$ ADP, $40 \mathrm{nM}$ GDP, $50 \mathrm{nM}$ ATP, $50 \mathrm{nM}$ GTP, $8 \mathrm{nM}$ NADH and $140 \mathrm{nM}$ acetyl-coA. (Concentrations shown are at spike-in and will be $\sim 10 \times$ more concentrated in the final reconstituted sample.)

\section{Liquid Chromatography}

Capillary columns were prepared as previously described. ${ }^{14}$ Briefly, macroporous frits were photopolymerized in place $5 \mathrm{~cm}$ from the end of the $75 \mu \mathrm{m}$ i.d. capillaries. Emitter tips were pulled to $3 \mu \mathrm{m}$ using a P-2000 laser-puller (Sutter Instrument, Novato, CA). $30 \mathrm{~cm}$ columns were packed using a slurry of $3 \mu \mathrm{m}$ Atlantis dC-18 aqueous reversed-phase particles in acetone.

The chromatographic system was setup as described in a previous work ${ }^{12}$ with an HPLC pump (Model 626, Waters, Milford, MA), a six-port injection valve and two flow-splitters. Sample injections $(15 \mathrm{~nL})$ onto the column were performed offline, i.e. by disconnecting the column from the pumping system and inserting the column inlet into a sample reservoir pressurized to $500 \mathrm{psi}$, to minimize extra band broadening associated with conventional injection valves. Metabolites were eluted using a 65 min ternary gradient. Mobile phase (MP) A was $20 \mathrm{mM}$ formic acid, $\mathrm{pH} 2.7$; MP B was $20 \mathrm{mM}$ ammonium formate, $\mathrm{pH}$ 6.5; and MP C was acetonitrile. The gradient profile was as follow (time, $\% \mathrm{~A}, \% \mathrm{~B}, \% \mathrm{C}$ ): $0 \mathrm{~min}, 100 \%, 0 \%, 0 \% ; 5 \mathrm{~min}, 100 \%$, $0 \%, 0 \%$; $10 \mathrm{~min}, 0 \%, 100 \%, 0 \%$; $18 \mathrm{~min}, 0 \%, 80 \%, 20 \%$; $23 \mathrm{~min}, 0 \%, 65 \%$ 35\%; $60 \mathrm{~min}, 0 \%$, $25 \%, 75 \%$; $65 \mathrm{~min}, 100 \%, 0 \%, 0 \%$.

\section{Mass Spectrometry}

Negative-ion mode electrospray mass spectra were obtained using an LCQ Deca XP Plus QITMS (Thermo-Electron, San Jose, CA) tuned for nucleotides (ATP). Spray voltage was -1.0 
$\mathrm{kV}$ and the capillary temperature was set to $150{ }^{\circ} \mathrm{C}$. Spectra were collected in full scan mode in a scan range of 50-1200 m/z. MS/MS spectra were obtained using isolation width $3 \mathrm{~m} / \mathrm{z}$, activation Q 0.25 , with collision energy optimized to each analyte.

\section{Data Processing}

MS Processor version 9.0 from Advanced Chemistry Development (Toronto, ON) was used for peak detection with the following parameters: MCQ threshold $=0.8$; smoothing window width $=3$; minimum full width at half maximum height $=3$; signal-to-noise $=5$. All features generated were manually screened to remove features resulted from dimers, multiple isotopes or adducts of sodium, chloride, and formate.

\section{Results and Discussion}

\section{Metabolite Standards}

Our work with standards was targeted at separation and detection of 25 metabolites in the glycolytic and tricarboxylic acid (TCA) cycle, which have a wide range of polarities, from polar glucose-6-phosphate (G6P) to non-polar flavin adenine dinucleotide (FAD). As shown in Figure 1, 19 metabolite standards were able to be separated; however isomer pairs F6P/G6P, 2-/3- phosphoglycerate (2/3PG), and citrate/isocitrate could not be differentiated because they co-eluted and had identical $\mathrm{m} / \mathrm{z}$. Although the small, phosphorylated sugars were less wellresolved than other metabolites as a result of low capacity, most of the metabolites yielded good resolution and peak shape.

Our system showed good sensitivity and reliability even though only $15 \mathrm{~nL}$ of sample was injected. Concentration detection limits were $7 \mathrm{nM}$ to $2 \mu \mathrm{M}$ for the glycolytic and TCA cycle intermediates, which is comparable to previous experiments on conventional HPLC ${ }^{22,23}$; however, because of the much smaller injection volume the mass detection limits were improved by 3 to 4 orders of magnitude to 0.1 to $33 \mathrm{fmol}$ (Table 1). Responses in signal-tonoise ratio (S/N) were linear from $0.3 \mu \mathrm{M}$ to $500 \mu \mathrm{M}$. Relative standard deviations (RSDs) of peak height for $10 \mu \mathrm{M}$ standards as a mixture were $7-18 \%$ from day to day $(\mathrm{n}=3)$ on same column and 9-21\% from column to column $(\mathrm{n}=3)$ as seen in Figure 2.

\section{Sample Preparation}

In metabolomic analysis, sample preparation is critically important. Instantaneous quenching of metabolism and efficient metabolite extraction are required due to the rapid turnover rate and small quantities of the metabolites of interest. ${ }^{24}$ Furthermore, when using electrospray ionization it is desirable to use as little salt as possible in sample preparation to decrease ion suppression.

Metabolic quenching by cold methanol is a popular method because it is able to deactivate metabolism within $100 \mathrm{~ms}^{24}$ and it enables the separation between extra- and intracellular metabolites. However this method can lead to significant loss of metabolites due to cell leakage if the methanol: water ratio is not optimized. ${ }^{25}$ Other methods involve treatment with perchloric acid or other acids/bases but these methods are not suitable for those metabolites that are not stable to extreme $\mathrm{pH}$ and oxidative/reductive media, such as ATP and NAD-related metabolites. Here we used snap freezing in liquid nitrogen as our quenching method.

Commonly used for plant and animal tissues,, 26 this method is able to quickly stop cell metabolism. After quenching, the islet was separated from media to avoid disturbance from secreted metabolites. To validate the quenching procedure, we analyzed the culture media with and without snap freezing in the presence of an islet. In this experiment, we did not detect any effect of freezing on metabolite content of the media $(n=3)$. Because metabolites that leaked into the media were below our detection limit, we could only calculate an upper limit to the 
amount of leakage that could occur. Based on the detection limits of the method, the volume of media islets were in when frozen, and the amount of metabolites present in extracts, we determined that leakage for all known metabolites detected must be less than 0.1 to $9.8 \%$ of their total islet content. Future work may be required to fully optimize this procedure. For example, other quenching methods may achieve faster arrest of metabolism.

Common extraction methods include cold or boiling organic solvents with buffers and acidic or alkaline extractions, with the latter evidently more suitable for metabolites stable to $\mathrm{pH}$ extremes. Extraction with boiling solvents is reported to be simple, fast, accurate and reliable. 27 This method however is not suitable for metabolites that are not stable at the extraction temperature, e.g., succinate has been reported to show poor recovery compared with cold solvent methods. ${ }^{28}$ In this study, we chose the cold methanol: water extraction method as our major focus is on the polar anions such as those found in the glycolytic and TCA pathways. No buffer was added during extraction to maximize sample simplicity for downstream electrospray. The 80:20 methanol: water ratio (v/v) was chosen according to a previous study on E. coli. 29

To verify this ratio and determine metabolite stability during extraction, we performed an isotope tracer spike-in test. ${ }^{29}$ In this test, metabolites labeled with a stable isotope are added to the sample prior to extraction and the signal for the standards is compared to the same standards injected directly. Decreases from the extracted sample would be indicative of sample loss during extraction. For our experiments, islet metabolites were labeled with ${ }^{13} \mathrm{C}$ by incubating islets in media containing $\mathrm{U}^{13} \mathrm{C}$-glucose allowing spiking of ${ }^{12} \mathrm{C}$ standards to be used for estimation of recovery. (All metabolites examined were fully labeled with ${ }^{13} \mathrm{C}$ glucose after the one-day incubation except ADP, GDP, ATP, GTP, NADH and acetyl-coA which were only partially labeled.) We compared the recovery of metabolite standards extracted by methanol: water ratios of 100:0, 80:20, and 50:50. As shown in Figure 3, the 80:20 ratio gave the highest recovery ratio (Figure 3 ).

The stability of analytes to extraction method was also validated by the same experiment. As shown in Figure 3, the ratios in peak height for all the tested compounds were close to 1 indicating little loss of metabolite during processing. Of the compounds tested, GTP and NADH showed the biggest loss of $\sim 14 \%$. Further confirming the stability of the metabolites, we were unable to detect ${ }^{12} \mathrm{C}$-labeled ADP, GDP, AMP, or GMP in the samples spiked with ${ }^{12} \mathrm{C}$-labeled ATP or GTP. These results also indicate that likely products of decomposition of ATP or GTP would not significantly affect measurement of nucleotide metabolites.

We also tested the stability of the single islet extracts stored at $-80^{\circ} \mathrm{C}$. Islet extracts were divided into 6 aliquots, dried and stored at $-80^{\circ} \mathrm{C}$. Aliquots were analyzed as duplicates every other day over an 11-day period. As shown in Figure 4, this analysis yielded RSDs of $4 \%$ to $17 \%$ for known compounds. The RSD is similar to assays of fresh standards over this period indicating that instability is not a factor in precision for this long of a time. For identified metabolites, average peak heights of day $7-11$ varied from $-12.6 \%$ to $+3.6 \%$, with a total average of $-5.4 \%$, of the peak heights of day $1-5$. Combined, these results indicate that freezedried samples are stable for at least 11 days.

\section{Islet Extract Analysis}

We analyzed 15 individual islets using the method with the QIT in full scan mode. A typical base peak chromatogram is shown in Figure 5B. Automated peak detection yielded more than 400 features which was reduced to 200 after removing signals that were due to multiple isotopes or adducts of sodium, chloride, and formate. Of these 200 presumed metabolites (other adducts or dimers are still possible among this group of features), we were able to identify 22 by comparing retention time and $\mathrm{m} / \mathrm{z}$ to standards. A detected peak was considered tentatively 
identified when $\mathrm{m} / \mathrm{z}$ was within $\pm 1 \mathrm{Da}$ and its retention time was within $2 \%$ compared with those found in standards. To further confirm the identification, LC-MS/MS runs targeting each of the 22 parent ions (there were 3 isomer pairs) performed on the islet extracts and the resulting MS/MS spectra compared to standards. Data demonstrating identification of NADH is shown in Figure 5. (Similar data for succinate are shown in supplemental Figure 1). All 25 compounds that had been tentatively identified were confirmed by this approach.

We examined the precision of analysis from a single islet and for different islets. RSDs of the identified compounds for different runs from the same islet were 9-23\% and from different islets were 10-25\% (Figure 2). Because the inter-islet consistency is comparable to that for intra-islet analysis, we conclude that the differences in islets and sample preparation are not a dominant source of variability.

The method consumes small amounts of the extract suggesting the potential for many interesting types of experiments. Single islets, which contain about 3000 cells, were reconstituted to a $20 \mu \mathrm{L}$ volume. Only $15 \mathrm{~nL}$ of this sample was used for analysis meaning that each injection corresponded to $\sim 2$ cells suggesting that the method has the sensitivity to perform single cell analysis, though improved sample preparation would be required to work at the single cell level. Because such a small fraction of the sample was used, another possibility is to perform multiple assays on an individual islet. As demonstrated above, this allows for reproducibility analysis on single islets. Perhaps more interesting is to use multiple assay conditions on the same sample to increase the number of metabolites detected. We were able to perform both anion exchange and reversed phase analysis on the same islet. In the anion exchange analysis, which is provided vastly different selectivity from the reversed phase separation, we found $\sim 250$ features, $58 \%$ which had different masses from those detected by reversed phase chromatography suggesting a significant expansion of metabolome coverage. (Sample chromatogram shown in Supplemental Figure 2.)

\section{Effects of Glucose}

To further evaluate the methodology, we compared the concentration of glycolytic and TCA cycle metabolites in single islets exposed to $3(n=6)$ or $20 \mathrm{mM}(n=6)$ glucose, the latter which results in enhanced glucose metabolism and insulin secretion. The results from this comparison are summarized in Figures 6, 7, and 8. Comparing our results with previous studies and known aspects of islet metabolism reveals that the method was able to detect expected changes in metabolites.

Glycolytic intermediates-Most of the glycolytic intermediates were unchanged (Figure 6) except for phosphoenolpyruvate (PEP) and pyruvate, suggesting that pyruvate kinase, which converts PEP to pyruvate may be partially limiting to glycolytic flux in agreement with recent modeling. ${ }^{30}$ The large increase in pyruvate is in agreement with a recent GC-MS study that analyzed the effect of glucose treatment on metabolites the $832 / 13 \beta$-cell line. ${ }^{19}$ The increase in pyruvate, along with lack of detection of significant lactate, is consistent with low lactate dehydrogenase activity (which would convert pyruvate to lactate) and monocarboxylate transporter activity (which would excrete pyruvate from the cell) in $\beta$-cells. ${ }^{31}$

TCA cycle intermediates-Almost all of the TCA cycle intermediates increased with elevated glucose (see Figure 6). This result suggests high mitochondrial activity and is consistent with the main fate of pyruvate to be transport into mitochondria for conversion to oxaloacetate or acetyl-CoA. We found for example that malate, succinate, citrate/isocitrate, acetyl-CoA, and succinyl-CoA increased $4.5 \pm 0.8_{3}, 8.3 \pm 1.9_{7}, 4.4 \pm 1.0_{8}, 7.7 \pm 1.1_{0}$, and 2.6 \pm 0.45 fold respectively in $20 \mathrm{mM}$ glucose compared to $3 \mathrm{mM}$ glucose ( $<<0.002$ ). Only $\alpha$ ketoglutarate did not have a substantial increase with glucose. Our results are mostly consistent 
with previous findings and known metabolism of islets or $\beta$-cell lines. ${ }^{31,} 32$ MacDonald $^{33}$ found a 2.0-fold increase in malate, a 1.5-fold increase in citrate/isocitrate, and a 1.7-fold increase in $\alpha$-ketoglutarate in cultured rat pancreatic islets when comparing islets incubated in 2 and $16.7 \mathrm{mM}$ glucose for $30 \mathrm{~min}$. That work also demonstrated a 1.5 -fold increase in acetylCoA and a 1.9-fold increase in succinyl-CoA in INS-1 832/13 cells incubated in 16.7 versus $1.5 \mathrm{mM}$ glucose for $30 \mathrm{~min}$. Other studies found similar effects such as a 6-fold increase in malate and a 2.2-fold increase in citrate in INS-1 cells treated with $20 \mathrm{mM}$ glucose for $30 \mathrm{~min}$. 32 Succinate was found to increase 1.4-fold when incubated in $16.7 \mathrm{mM}$ glucose for $1 \mathrm{~h}$ versus $2.8 \mathrm{mM}$ glucose, while malate increased 1.2 -fold. ${ }^{34}$ A recent metabolomic study ${ }^{19}$ using GCMS analysis of INS-1 cells found increases in citrate, fumarate, and malate in response to glucose stimulation that were similar to ours.

The strong increase in TCA intermediates is indicative of anaplerosis, which refers to the "filling up" of TCA cycle or increasing in intermediates. Anaplerosis is an intrinsic feature of $\beta$-cells and has been implicated in producing signals that couple glucose concentration to insulin secretion. ${ }^{17,} 31$ Interestingly, it has been shown that carbons are lost from the TCA cycle through $\alpha$-ketoglutarate to produce glutamate, a potential coupling factor for insulin secretion. ${ }^{35}$ This metabolic pathway may explain our observation of no change in $\alpha$ ketoglutarate even though other TCA intermediates increased.

Nucleotides-Nucleotide changes (Figure 7) were largely in agreement with previous studies. It is well known that the ATP/ADP ratio increases in islets with elevations of glucose. We found an increase of 7.2 \pm 1.97 which is comparable to the 4.6-fold increase found in purified $\beta$-cells incubated in $10 \mathrm{mM}$ glucose versus $1 \mathrm{mM}$ glucose for $1 \mathrm{~h} .{ }^{36}$ Other studies have shown an approximate 3-fold increase in ATP/ADP ratio when incubated in similar conditions. ${ }^{37-39}$ The GTP/GDP ratio has also been reported to increase upon treatment with elevated glucose concentration. ${ }^{37,40}$ Our observation of a $1.6 \pm 0.45$ fold increase is comparable to a previous study where the GTP/GDP ratio was found to increase 1.5 fold after 30 min perfusion with 5 versus $28 \mathrm{mM}$ glucose ${ }^{40}$ but less than the 5.5 fold increase found for islets incubated in 3 versus $20 \mathrm{mM}$ glucose for $1 \mathrm{~h}^{37}$. We also found that the total amount of nucleotides increased, which is interesting because the synthesis of these compounds is generally considered to be too slow to occur over the time scale of this experiment. The increase in total nucleotides however corresponds well to previous studies of islets that showed that increases in the sum of ATP and GTP were much higher than decreases in the sum of ADP and GDP. 37

An interesting aspect of the method is that because the QIT-MS is operated in full scan mode, it allows unidentified features to be tracked. Among the unidentified features, $\sim 40$ increased more than 2-fold with $20 \mathrm{mM}$ glucose, $\sim 10$ decreased more than 2-fold with $20 \mathrm{mM}$ glucose, and another 50 varied less than 2-fold with glucose (Figure 9). Identification of these features may be of interest for deeper studies of the metabolome. Such identification will largely rely on comparison to standards as illustrated in Figure 5.

Overall our results are consistent with previous reports obtained using various assay methods. Although some quantitative differences between our results and previous studies were found, they may be attributed to differences in cell types (mouse versus rat islets or INS-1 cells) and incubation conditions. The differences in selectivity of the LC-MS method and the enzyme assays that have typically been used may also contribute to some of the differences observed. The power of the LC-MS method is the ability to measure all of the compounds simultaneously in single islets. As most studies have only observed a few of these compounds at once and been limited to studying cell lines, this measurement method provides a unique opportunity to study islet metabolism. 


\section{Conclusions}

In this work, we demonstrated a microscale method for metabolomic analysis from sample preparation to separation and detection using capillary LC-ESI-MS in negative mode. Limits of detection for targeted metabolite standards from glycolysis/TCA cycle intermediates were in the concentration range of low nanomolar to low micromolar indicating suitable sensitivity for detection of various intracellular metabolites in a complex biological system, such as single islet. Further improvements in detection limit should be possible by using reaction monitoring (i.e., MS-MS) modes of detection; however, the use of the QIT-MS in full scan mode allowed both targeted compounds to be monitored and unknowns. The reproducibility of the method was sufficient to detect large, relative changes in metabolite concentrations associated with fuel changes without use of internal standards. The minimal amount of sample used suggests applications such as single cell analysis and performing multiple assays on the same islets. Future work will be devoted to exploring improved separation conditions for the polar analytes. In particular, LC conditions that allow on-column preconcentration will substantially improve the sensitivity. Better LC conditions, plus tests for other compounds should greatly expand the number of metabolites detectable by this method.

\section{Acknowledgements}

This work was supported by NIH grant DK046960 to R.T.K. C.F.B. received support from the Michigan Metabolomics and Obesity Center.

\section{Literature Cited}

1. Fiehn O. Plant Mol Biol 2002;48:155-171. [PubMed: 11860207]

2. Hollywood K, Brison DR, Goodacre R. Proteomics 2006;6:4716-4723. [PubMed: 16888765]

3. Rochfort S. J Nat Prod 2005;68:1813-1820. [PubMed: 16378385]

4. Griffin JL, Bollard ME. Curr Drug Metab 2004;5:389-398. [PubMed: 15544433]

5. Dunn WB, Bailey NJ, Johnson HE. Analyst 2005;130:606-625. [PubMed: 15852128]

6. Lenz EM, Wilson ID. J Proteome Res 2007;6:443-458. [PubMed: 17269702]

7. Want EJ, Cravatt BF, Siuzdak G. Chembiochem 2005;6:1941-1951. [PubMed: 16206229]

8. Want EJ, Nordström A, Morita H, Siuzdak G. J Proteome Res 2007;6:459-468. [PubMed: 17269703]

9. Villas-Bôas SG, Mas S, Akesson M, Smedsgaard J, Nielsen J. Mass Spectrom Rev 2005;24:613-646. [PubMed: 15389842]

10. Edwards JL, Kennedy RT. Anal Chem 2005;77:2201-2209. [PubMed: 15801754]

11. Kennedy RT, Jorgenson JW. Anal Chem 1989;61:1128-1135.

12. Edwards JL, Edwards RL, Reid KR, Kennedy RT. J Chromatogr A 2007;1172:127-134. [PubMed: 17961581]

13. Karas M, Bahr U, Dülcks T. Fresenius J Anal Chem 2000;366:669-676. [PubMed: 11225778]

14. Haskins WE, Wang Z, Watson CJ, Rostand RR, Witowski SR, Powell DH, Kennedy RT. Anal Chem 2001;73:5005-5014. [PubMed: 11721892]

15. Deeney JT, Prentki M, Corkey BE. Semin Cell Dev Biol 2000;11:267-275. [PubMed: 10966860]

16. Prentki M. Eur J Endocrinol 1996;134:272-286. [PubMed: 8616523]

17. MacDonald M, Fahien L, Brown L, Hasan N, Buss J, Kendrick M. Am J Physiol Endocrinol Metab 2005;288:E1-E15. [PubMed: 15585595]

18. Fridlyand LE, Philipson LH. Curr Diabetes Rev 2006;2:241-259. [PubMed: 18220630]

19. Fernandez C, Fransson U, Hallgard E, Spégel P, Holm C, Krogh M, Wårell K, James P, Mulder H. J Proteome Res 2008;7:400-411. [PubMed: 18062666]

20. Ronnebaum SM, Ilkayeva O, Burgess SC, Joseph JW, Lu D, Stevens RD, Becker TC, Sherry AD, Newgard CB, Jensen MV. J Biol Chem 2006;281:30593-30602. [PubMed: 16912049] 
21. Roper MG, Shackman JG, Dahlgren GM, Kennedy RT. Anal Chem 2003;75:4711-4717. [PubMed: 14674445]

22. Mashego MR, Wu L, Van Dam JC, Ras C, Vinke JL, Van Winden WA, Van Gulik WM, Heijnen JJ. Biotechnol Bioeng 2004;85:620-628. [PubMed: 14966803]

23. Bajad SU, Lu W, Kimball EH, Yuan J, Peterson C, Rabinowitz JD. J Chromatogr A 2006;1125:7688. [PubMed: 16759663]

24. de Koning W, van Dam K. Anal Biochem 1992;204:118-123. [PubMed: 1514678]

25. Bolten CJ, Kiefer P, Letisse F, Portais JC, Wittmann C. Anal Chem 2007;79:3843-3849. [PubMed: 17411014]

26. Bundy JG, Spurgeon DJ, Svendsen C, Hankard PK, Osborn D, Lindon JC, Nicholson JK. FEBS Lett 2002;521:115-120. [PubMed: 12067738]

27. Gonzalez B, François J, Renaud M. Yeast 1997;13:1347-1355. [PubMed: 9392079]

28. Maharjan RP, Ferenci T. Anal Biochem 2003;313:145-154. [PubMed: 12576070]

29. Kimball E, Rabinowitz JD. Anal Biochem 2006;358:273-280. [PubMed: 16962982]

30. Jiang N, Cox RD, Hancock JM. Mamm Genome 2007;18:508-520. [PubMed: 17514510]

31. Ishihara H, Wollheim CB. IUBMB Life 2000;49:391-395. [PubMed: 10902570]

32. Schuit F, De Vos A, Farfari S, Moens K, Pipeleers D, Brun T, Prentki M. J Biol Chem 1997;272:18572-18579. [PubMed: 9228023]

33. MacDonald M. J Biol Chem 2007:282.

34. Alarcon C, Wicksteed B, Prentki M, Corkey BE, Rhodes CJ. Diabetes 2002;51:2496-2504. [PubMed: 12145163]

35. MacDonald MJ. Biochim Biophys Acta 2003;1619:77-88. [PubMed: 12495818]

36. Detimary P, Dejonghe S, Ling Z, Pipeleers D, Schuit F, Henquin JC. J Biol Chem 1998;273:3390533908. [PubMed: 9852040]

37. Detimary P, Van den Berghe G, Henquin JC. J Biol Chem 1996;271:20559-20565. [PubMed: 8702800]

38. Nilsson T, Schultz V, Berggren PO, Corkey BE, Tornheim K. Biochem J 1996;314:91-94. [PubMed: 8660314]

39. Giroix MH, Agascioglu E, Oguzhan B, Louchami K, Zhang Y, Courtois P, Malaisse WJ, Sener A. Biochim Biophys Acta 2006;1757:773-780. [PubMed: 16782044]

40. Meglasson MD, Nelson J, Nelson D, Erecinska M. Metabolism 1989;38:1188-1195. [PubMed: 2687638] 




Figure 1.

Separation of metabolite standards (10 $\mu \mathrm{M}$ of each metabolite) found in glycolysis and TCA cycle. Reconstructed ion chromatograms (RICs) are shown in retention time (RT) segments (A) $8.5-12.5 \mathrm{~min}$ and (B) $17-32 \mathrm{~min}$. The column was $35 \mathrm{~cm}$ long by $75 \mu \mathrm{m}$ i.d. packed to 30 $\mathrm{cm}$ with Atlantis dC-18 $3 \mu \mathrm{m}$ particles with an integrated nanospray tip of $2 \mu \mathrm{m}$ diameter. F6P/ G6P, fructose 6-phosphate/glucose 6-phosphate; 1,3-BPG, 1,3-biphosphoglycerate; FBP, fructose 1,6-biphosphate; G3P, glyceraldehyde 3-phosphate; PEP, phosphoenolpyruvate; OAA, oxaloacetate; Pyr, pyruvate; $\alpha \mathrm{KG}, \alpha$ ketoglutarate; $2 / 3$ PG, 2-/3- phosphoglycerate. 


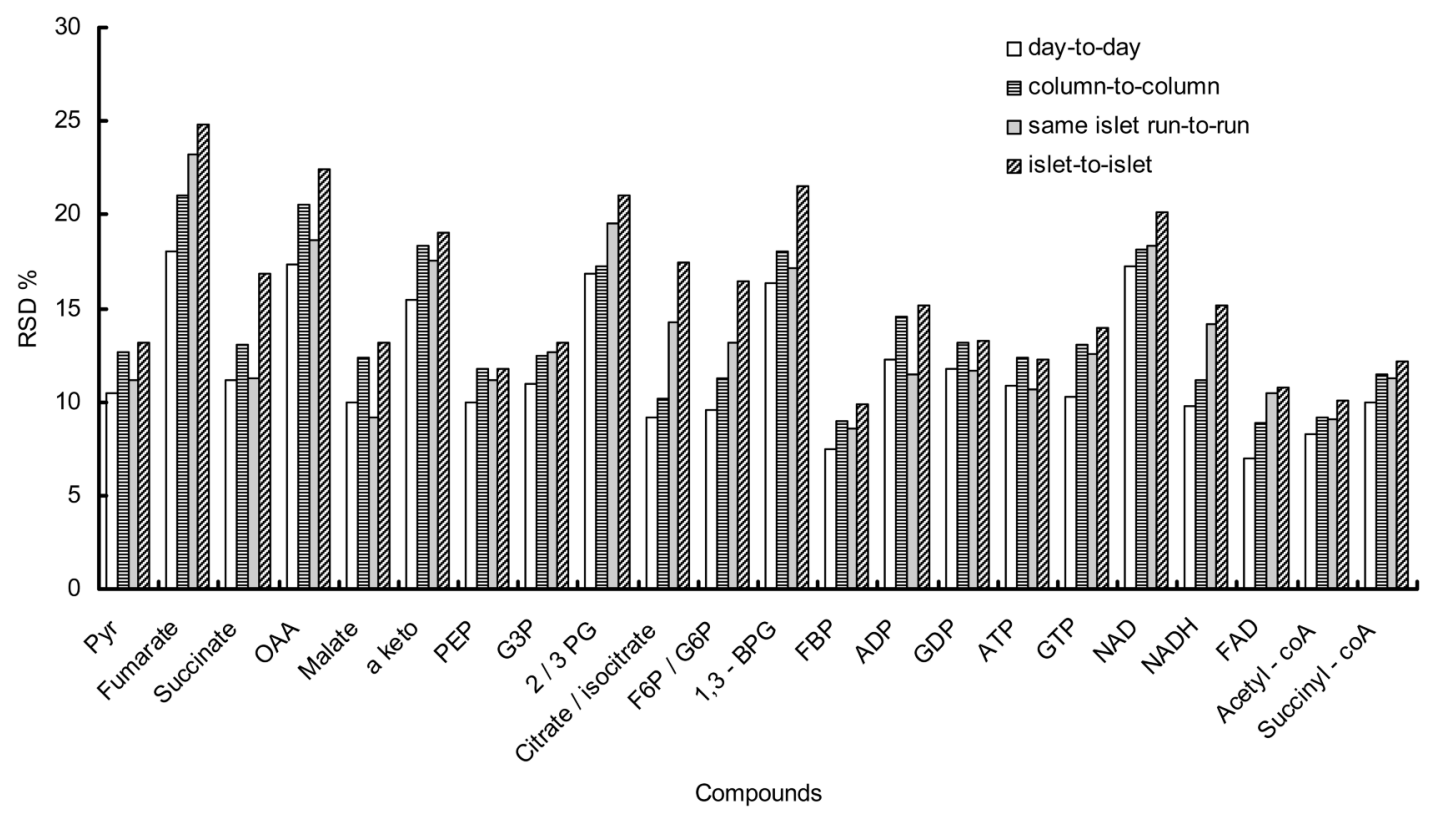

Figure 2.

Reproducibility of $10 \mu \mathrm{M}$ metabolite standards signals on same column from day to day and from column to column. Reproducibility of identified metabolites from same islet extract from run to run and from different islets is also shown. For all cases samples were analyzed in triplicate. 


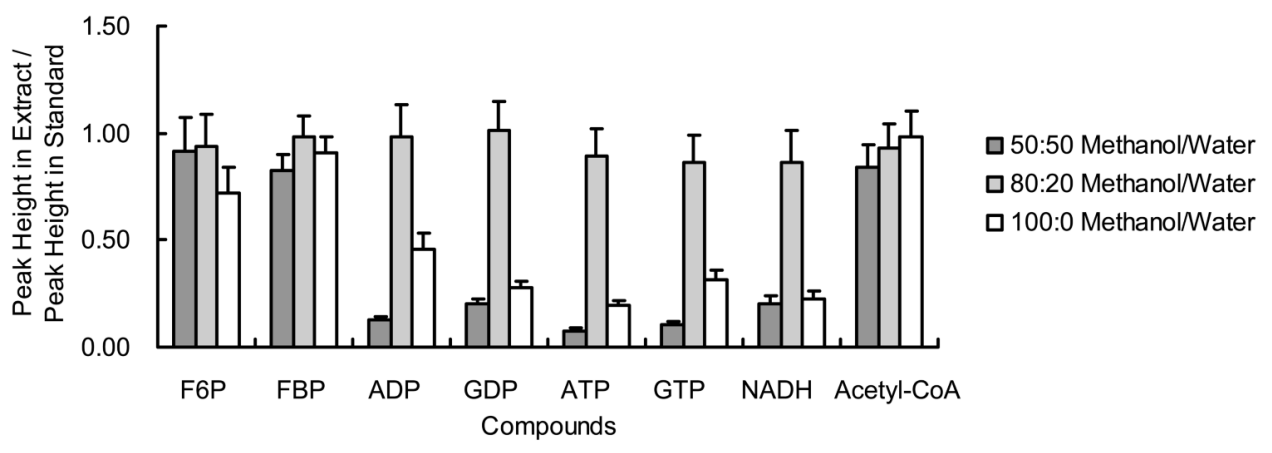

Figure 3.

Recovery of spiked metabolites extracted respectively by 100:0, 80:20, and 50:50 methanol: water. Fully ${ }^{13} \mathrm{C}$-labeled single islet was extracted using solvent spiked with ${ }^{12} \mathrm{C}$ metabolites. The signal of the spiked ${ }^{12} \mathrm{C}$ metabolites obtained in the resulting extract is compared with the signal anticipated assuming no metabolite loss $(n=3)$. Error bars represent standard deviations. 


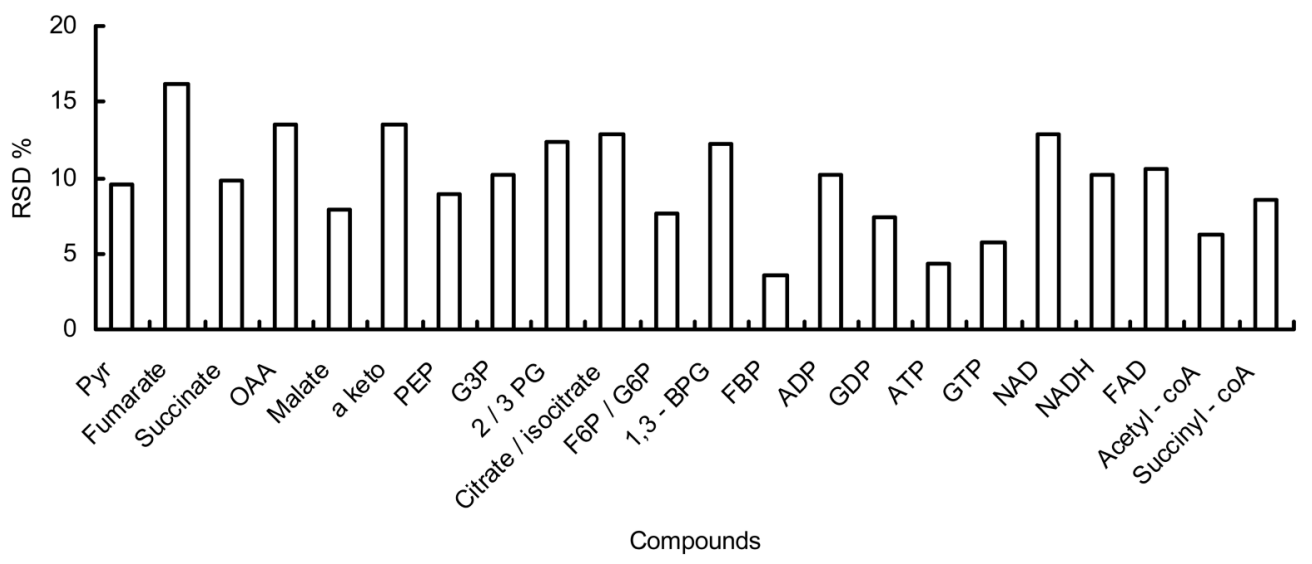

Figure 4.

Test of sample stability. Islet extracts from the same islet were stored freeze-dried under -80 ${ }^{\circ} \mathrm{C}$ and analyzed over time. RSDs for identified metabolites over 11 days (samples were tested every other day and $n=2$ for each day) were within $17 \%$. 


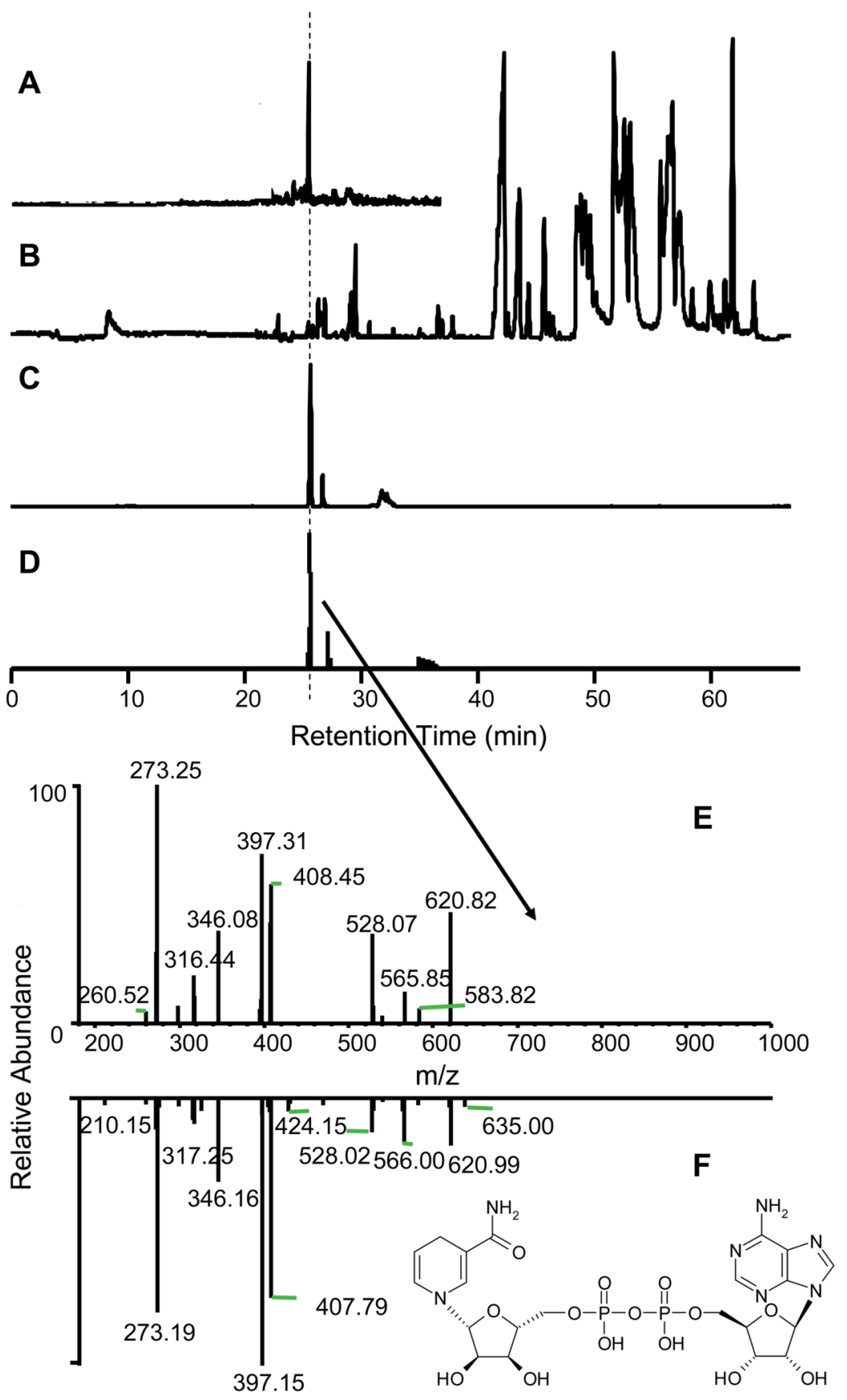

Figure 5.

Sample identification of NADH from single islet extracts. RIC MS $m / z 664$ (A) from base peak islet extract (B) was first retention time matched to RIC MS NADH $\mathrm{m} / z 664$ from standard (C). MS/MS of $m / z 664$ was performed on the islet extracts and the RIC of $m / z 664 \rightarrow 273+$ $397+408$ is shown (D) with same retention time (as indicated by the dotted line). The MS/ MS fragmentation of $m / z 664$ from extract (E) matched standard NADH MS/MS m/z 664 spectrum $(\mathrm{F})$. 


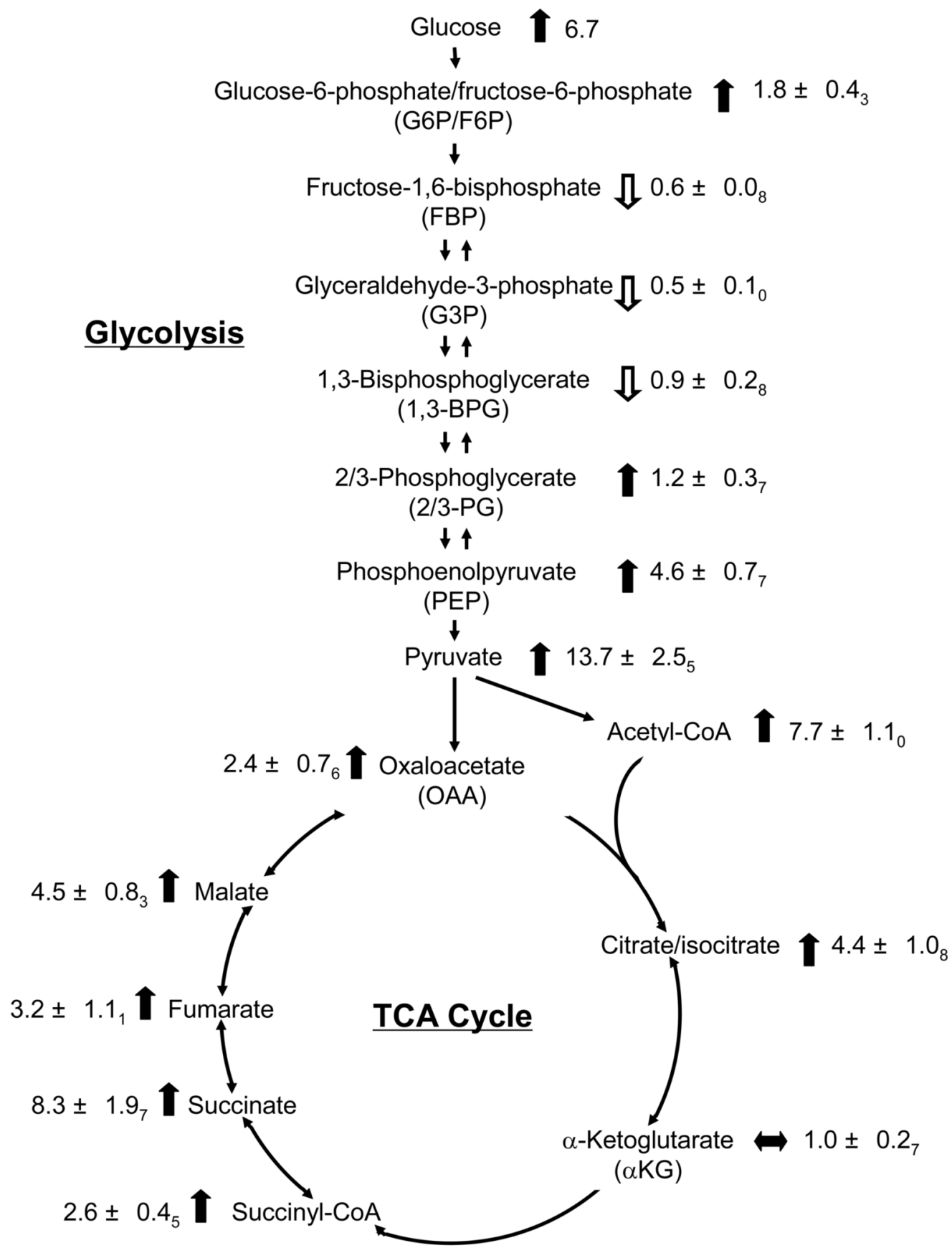

Figure 6.

Changes in glycolytic and TCA metabolites in a single islet stimulated by glucose. Islets were cultured in 3,20 mM glucose before extraction. Ratio of identified metabolites concentrations in $20 \mathrm{mM}$ glucose over those in $3 \mathrm{mM}$ glucose varied from 0.5 (G3P) to 13.7 (pyr), with empty arrows indicating decrease and filled arrows indicating increase with the increase of glucose concentration. 


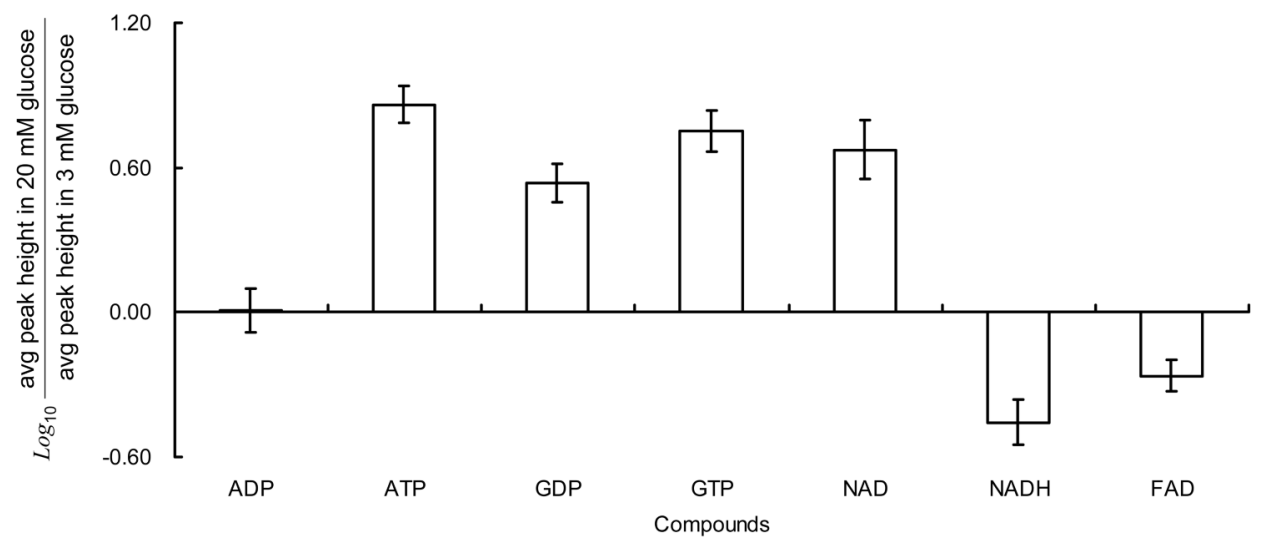

Figure 7.

Changes of energy transfer metabolites in glycolysis and TCA cycle with glucose stimulation. Islets were cultured in 3, $20 \mathrm{mM}$ glucose before extraction. Ratio of concentrations in $20 \mathrm{mM}$ glucose over those in $3 \mathrm{mM}$ glucose varied from 0.3 (NADH) to 7.3 (ATP), with downwardfacing bars indicating decrease and upward-facing bars indicating increase with the increase of glucose concentration. Error bars represent \pm 1 standard deviation. 


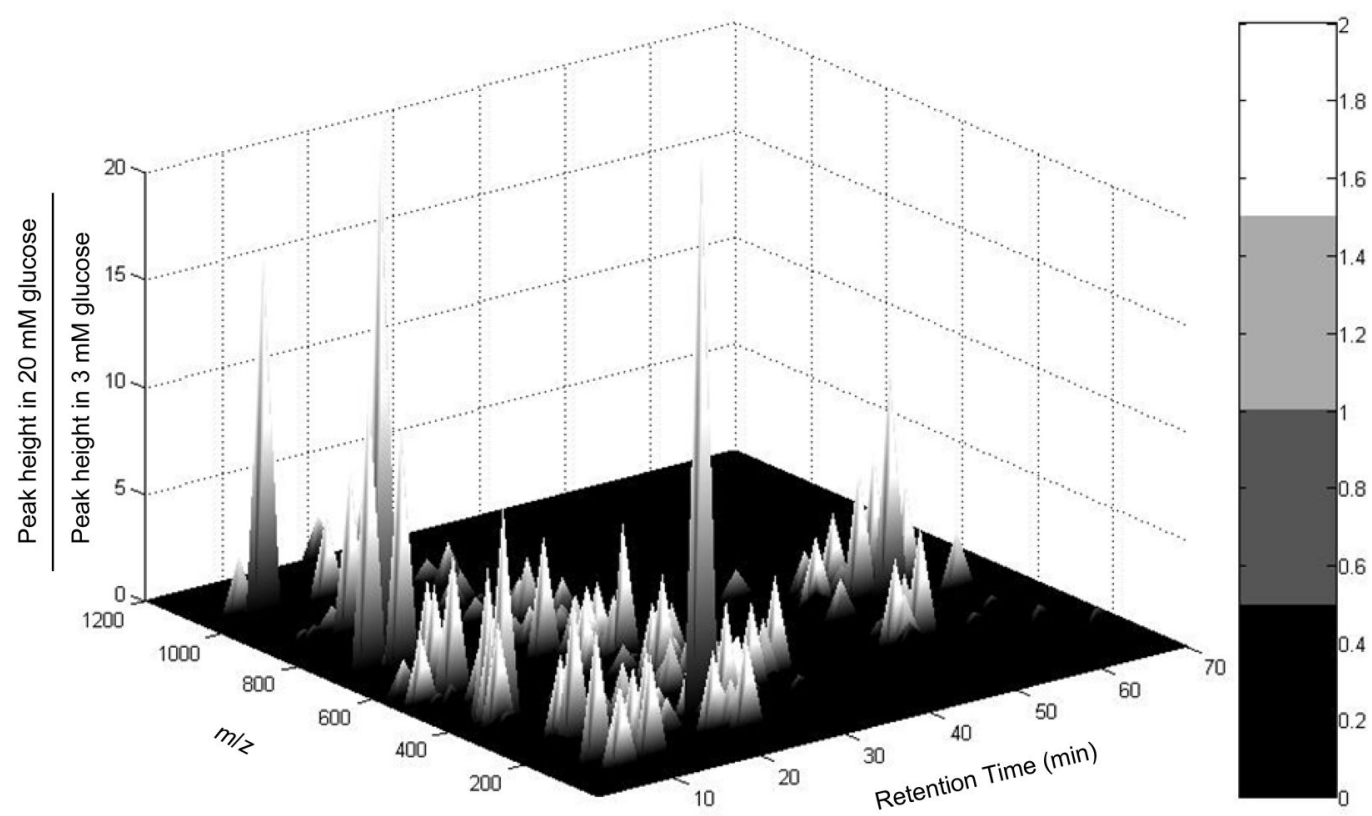

Figure 8.

Three-dimensional bar graph showing changes of unknown metabolites with glucose stimulation. Both retention time and $\mathrm{m} / \mathrm{z}$ were shown for each feature detected by MS Processor software. 40 features increased more than 2 fold with $20 \mathrm{mM}$ glucose, $\sim 10$ increased more than 2 fold with $3 \mathrm{mM}$ glucose, and another 50 varied less than 2 fold with glucose. 
Table 1

Summary of detection of metabolites from glycolysis/TCA cycle intermediates and their changes in single islet with glucose stimulation. $\mathrm{m} / \mathrm{z}$ for peaks were rounded to nearest integer for clarity.

\begin{tabular}{|c|c|c|c|c|}
\hline Compound & $\boldsymbol{m} / \mathbf{z}$ found & $\mathbf{t}_{\mathbf{R}}(\mathbf{m i n})$ & $\mathbf{L O D}(\mathbf{n M})$ & Change with glucose stimulation Avg (20 mM)/avg (3 mM) \\
\hline Pyruvate & 87 & 9.8 & 160 & $13.7 \pm 2.5_{5}$ \\
\hline Fumarate & 115 & 11.1 & 551 & $3.2 \pm 1.1_{1}$ \\
\hline Succinate & 117 & 17.1 & 1100 & $8.3 \pm 1.9_{7}$ \\
\hline Oxaloacetate & 131 & 9.7 & 2250 & $4.4^{2} \pm 0.7_{6}$ \\
\hline Malate & 133 & 10.8 & 870 & $1.0 \pm 0.8_{3}$ \\
\hline$\alpha$ ketoglutarate & 146 & 11.0 & 605 & $4.6 \pm 0.7_{7}$ \\
\hline PEP & 167 & 9.6 & 830 & $0.5 \pm 0.1_{0}$ \\
\hline G3P & 169 & 9.4 & 103 & $1.2 \pm 0.3_{7}$ \\
\hline 2/3 PG & 184 & 31.5 & 1880 & $4.4 \pm 1.0_{8}$ \\
\hline Citrate/isocitrate & 192 & 11.1 & 145 & $1.8 \pm 0.4_{3}$ \\
\hline F6P/G6P & 260 & 9.1 & 60 & $0.9 \pm 0.2_{8}$ \\
\hline 1,3 - BPG & 265 & 9.2 & 131 & $0.6 \pm 0.0_{8}$ \\
\hline FBP & 340 & 9.2 & 140 & $1.0 \pm 0.2_{2}$ \\
\hline ADP & 427 & 10.7 & 195 & $3.4 \pm 0.6_{5}$ \\
\hline GDP & 443 & 12.0 & 350 & $7.3 \pm 1.2_{7}$ \\
\hline ATP & 507 & 9.6 & 30 & $5.6 \pm 1.1_{2}$ \\
\hline GTP & 521 & 9.0 & 300 & $4.7 \pm 1.3_{4}$ \\
\hline NAD & 662 & 23.1 & 413 & $0.3 \pm 0.0_{7}$ \\
\hline NADH & 664 & 25.1 & 25 & $0.5 \pm 0.0_{8}$ \\
\hline FAD & 785 & 31.2 & 7 & $7.7 \pm 1.1_{0}$ \\
\hline Acetyl - coA & 808 & 29.5 & 14 & $2.6 \pm 0.4_{5}$ \\
\hline Succinyl - coA & 866 & 27.8 & 18 & \\
\hline & & & & \\
\hline
\end{tabular}

